\title{
Interference Cancellation Schemes in UWB Systems used in Wireless Personal Area Network based on Wavelet based Pulse Spectral shaping and Transmitted Reference UWB using AWGN Channel Model
}

\author{
Bikramaditya Das \\ Department of Electrical \\ Engineering \\ National Institute of Technology, \\ Rourkela -769008, India
}

\author{
Ch.Sasmita Das \\ Department of Electrical \\ Engineering \\ National Institute of Technology, \\ Rourkela -769008, India
}

\author{
Susmita Das, Member, IEEE \\ Department of Electrical \\ Engineering \\ National Institute of Technology, \\ Rourkela -769008, India
}

\begin{abstract}
For high data rate ultra wideband communication system, performance study of mutual interference such as suppression of interference signal by performing pulse shaping from UWB to other narrowband system using wavelet technique and reduction of interference from other narrowband system to UWB system in conjunction with Transmitted-Reference (TR-UWB) signaling scheme with adaptive receivers is attempted in this paper. The TRUWB signal using wavelet is based on multi-resolution technique to avoid its interference to other wireless systems. Ultra Wideband (UWB) wireless systems supposed to coexist with other spectrally overlapping wireless systems such as narrowband interference (NBI), multiple NBI and wideband interference allocated from $3.1 \mathrm{GHz}$ to 10.6GHz. So suppression of UWB from other system can be performed by using Eigen value decomposition using wavelet technique. Mutual interference degrades the performance of the TRUWB system using auto-correlation receivers (AcR). In this paper adaptive receiver (using notch filter) is used for detection and suppression of mutual interference (narrowband and wideband signal) which do not require modification of the narrowband system. The bit error rate performances are investigated using MATLAB simulation under AWGN channel model. At 10-2 BER floor, TRUWB using adaptive receiver with NBI simulations under AWGN provides a more than of $6 \mathrm{~dB}$ SNR improvement than that of TR using AcR receiver with NBI. The performance degrades when coexistence of multiple NBI and wideband interference wireless system with UWB system is applied using the same receiver.
\end{abstract}

Keywords- TR-UWB, AWGN Channel Model, NBI, multiple NBI , wideband interference ,Bit Error Rate .

\section{INTRODUCTION}

Ultra Wideband Radio (UWB) is an emerging technology with big promise in imaging systems, vehicular radar systems, and communication and measurement systems. UWB technology has for many years been used in radar and military communications but has not been allowed on the open market prior to 2002. In April 2002, the federal communication commission (FCC) [1] lifted the restriction on the use of UWB technology for non-military applications. Since then, more industries have started developing UWB systems. UWB systems send information with extremely short duration pulses, therefore allowing high speed data communication and diversity against multipath. Unlike traditional narrowband systems, UWB generates short pulses and uses these pulses for data modulation. Therefore, UWB is alternatively referred to as impulsive, carrierless or baseband transmission. However, it has now been realized that UWB does not have to be impulsive or carrierless [2]. This is because the FCC only defines UWB as a signal that occupies more than 500 $\mathrm{MHz}$ in the 3.1-10.6 GHz spectral mask. UWB communication systems use spectrum which is already allocated to other wireless systems, and so interference by and into UWB communication signals have been the subject of intense research interest; because reliable operation of these systems are highly sensitive to interference signals in the same band. UWB coexistence and interference issues are very important. Up to now, most investigations of coexistence issues concern the interference of UWB devices on existing services such as UMTS, GPS WLAN and GSM. But recently, research progress shows that mitigation of interference by UWB signals are possible by using different pulse shapes, modulation techniques and whitening and shaping of the power spectral density(PSD) [3], [4], [5]. There also exists some publications considering the impact of existing systems interference on UWB systems [6], [7]. But only in few publications, for example in [8], [9], interference mitigation techniques are considered. The authors in [8] have presented the use of multi-carrier type transmission pulse and template waveforms to mitigate narrow band interference. However, the use of simple correlator at receiver won't perform well in the multipath condition. Feedback loop mechanism has also been proposed to enhance the signal-to-noise ratio of reference pulses in a conventional TR receiver [9], however the mechanism was tested with sinusoidal interference model. In this paper, TR-UWB system performance is studied in the presence of strong narrowband, multiple NBI and wideband interference. To reduce the effects of interference by and into UWB signals, a new method is proposed in conjunction with a multi-carrier type transmission pulse technique.

The rest of the paper is organized as follows. Section 2 discusses Eigen value decomposition model for suppression of intrference signal from UWB to other narrowband system using wavelet technique. In Section 3, we study the system model of TR-UWB. Suppression of interference from single NBI, multiple NBI and wideband interference to UWB system with adaptive receiver is 
presented in Section 4. Simulation results are discussed in Section 5. Section 6 concludes the paper.

\section{INTERFERENCE CANCELLATION SCHEMES}

\subsection{Interference (NBI, WBI) suppression} based on UWB Pulse design using wavelet

\section{(Eigen value decomposition model)}

The algorithm for the UWB pulse design first presented in $[9$, 12]. Here the brief summary of this algorithm is introduced necessarily. Firstly, a desired frequency mask H(f) is given.

$$
H(f)= \begin{cases}1 & f_{L}<f<f_{H} \\ 0 & \text { elsewhere }\end{cases}
$$

where $\mathrm{fL}=3.1 \mathrm{GHz}, \mathrm{fH}=10.6 \mathrm{GHz}$. Simply we can get the inverse Fourier transform of $\mathrm{H}(\mathrm{f})$.

$$
h(t)=2 f H \operatorname{sinc}(2 f H t)-2 f L \operatorname{sinc}(2 f L t)
$$

The mask is equivalent to a frequency response of a filter.

So the UWB pulses $s(t)$ can be generated by filtering.

$$
\lambda s(t)=\int_{-\infty}^{\infty} s(\tau) h(t-\tau) d \tau
$$

Where $\lambda$ is an attenuation factor, $\mathrm{h}(\mathrm{t})$ is the inverse Fourier transform of $\mathrm{H}(\mathrm{f})$.

We wish to design a pulse $\mathrm{p}(\mathrm{t})$ that is time-limited.

$$
\mathrm{s}(\mathrm{t})=0, \quad|\mathrm{t}|>\mathrm{Tm} / 2
$$

Clearly, Tm is the time duration of UWB pulse. By sampling at a rate of $\mathrm{N}$ samples per pulse period $\mathrm{Tm}$, (2) can be expressed as follows:

$$
\lambda s[n]=\sum_{m=-N / 2}^{N / 2} s(m) h(n-m), n=-N / 2 \ldots \ldots . . N / 2
$$

where $\mathrm{n}$ and $\mathrm{m}$ are integer values. Equation (5) can be expressed in vector form as follows:

$$
\lambda \mathrm{s}=\mathrm{Hs}
$$

where vector s represents the discretized UWB pulse, $\mathrm{H}$ is a

real Hermitian Toeplitz matrix. Figure. 3 UWB pulse generated by using the eigenvector corresponding to the largest eigenvalue of $\mathrm{H}$. Here, $\mathrm{N}=128, \mathrm{Tm}=1 \mathrm{~ns}$. The power spectral density (PSD) of the pulse is depicted in the same figure. The frequency spectrum and power spectral density (PSD) if the UWB pulse can be calculated by

$$
s(f)=\frac{T_{m}}{N} \sum_{n=-N / 2}^{N / 2} s[n] e^{-j 2 \pi f \frac{n T_{m}}{N}}
$$

Which gives the same result as wavelet pulse design method.

$$
s(t)=\sum w_{f}\left(n f_{s}\right) \psi\left(\frac{t}{n f_{s}}\right)
$$

Where $\mathrm{n}$ is any integer, pulse repetition rate $f_{s}=400 \mathrm{MHz}$, $w_{f}\left(n f_{s}\right)_{\text {is known as wavelet coefficient and }} \psi\left(\frac{t}{n f_{s}}\right)$ represents sub carrierswith pulse width $1 \mathrm{~ns}$.

Suppose that the major power of the narrow-band interference is concentrated on its center frequency f0 [10]. So the optimal scheme is to form a zero point of the PSD of UWB pulse at the frequency f0. We can obtain the desired pulse as follows [9].

\subsubsection{Single NBI interference mitigation}

STEP-1- Partition the UWB band into two bands: $\left(f_{L}, f_{M}\right)$ and $\left(f_{N}, f_{H}\right)$. Note that $f_{N}>f_{M}$ is necessary.

STEP-2- In each band, use the eigenvalue decomposition method to generate two sub-pulses $s_{1}(t)$ and $s_{2}(t)$. Meanwhile, adjust $f_{M}, f_{N}$ and pulse amplitude to make $s_{1}(t)$ and $s_{2}(t)$ not only meet the FCC spectral mask, but also let $S_{1}\left(f_{0}\right)=-S_{2}\left(f_{0}\right)$. Here, $S_{1}\left(f_{0}\right)$ and $S_{2}\left(f_{0}\right)$ denote the frequency spectrums of $s_{1}(t)$ and $s_{2}(t)$.

STEP-3- Superpose the two sub-pulses to generate the desired UWB pulse. Clearly the PSD (power spectral density) of UWB pulse has a zero point at $f_{0}$. Figure. 3 shows the PSD of the UWB pulse suppressing single narrow-band interference.

\subsubsection{Multiple NBI interference mitigation}

In this section, we assume there are over three narrow-band interferences located in the UWB band. If we still use the method in [9], the number of sub-bands will be over four. After the simulation, we found that the lobe of the pulse becomes larger with the increment of the sub-band number. So we have to limit the number of the subband not more than three. Under this limitation, we present our new method to deal with the multiple narrow-band interference situations. This method is simple, effective and easy-implemented. The main steps are as follows:

STEP-1- Partition the UWB band into two bands: $\left(f_{L}, f_{M}\right)$ and $\left(f_{M}, f_{H}\right)$, then use the eigenvalue decomposition method to generate two pulse $s_{1}(t), s_{2}(t)$ respectively. Without loss of generality, we suppose that the narrow-band interferences all locate in the lower band $\left(f_{L}, f_{M}\right)$. So at the receiver, $s_{l}(t)$ should be further processed to mitigate the narrow-band interferences.

STEP-2- We obtain the desired pulse from $s(t)=s_{I}(t)+s_{2}(t)$. Figure.4 shows the waveform of $s_{3}(t)$, and the PSD of the composed pulse, here we choose $f_{M}=5.9 \mathrm{GHz}$.

STEP-3- At the receiver, we use a doublet pulse consisting of two received pulses $s_{l}(t)$ separated from each other by $T_{g}$ time and with opposite amplitudes [13]. $T_{g}$ represents the delay time. The processed pulse $s_{d}(t)$ can be expressed as:

$$
s_{d}(t)=\frac{1}{\sqrt{2}}\left(s_{1}(t)-s_{1}\left(t-T_{g}\right)\right)
$$

The spectral amplitude of such a pulse can be computed as:

$$
\left|S_{d}(f)\right|^{2}=2\left|S_{1}(f)\right|^{2} \sin ^{2}\left(\pi f T_{g}\right)
$$


Note that the spectrum has nulls at frequencies at $f=k T_{g}$ for any integer $\mathrm{k}$. By adjusting $\mathrm{T}_{\mathrm{g}}$, we can easily remove the interferences in the lower band. Figure. 4 shows the spectral characteristic clearly.

\subsubsection{Wideband interference mitigation}

In this section, we extend our method to the wide-band interference mitigation. Commonly, the UWB pulse is applied in the WPAN (wireless personal area network). It is possible that the UWB systems coexist with several narrow-band devices and wide-band devices simultaneously. In this situation, the process is a little more complicated than two sub-bands mode discussed in the last section. We will divide the UWB band into three sub-bands. We suppose that the interference has a bandwidth from $f I_{L}$ to $f I_{\mathrm{H}}$, note that $\left(f I_{\mathrm{L}}, f I_{\mathrm{H}}\right)$ locates in the middle of the UWB band. The wide-band systems employ the multiple carries; the main transmitted power is concentrated on the carriers, so our main idea remains to generate nulls at each frequency of the carrier. The difference between this mode and two sub-bands mode is the delay time $\mathrm{Tg}$ that could be longer according to the frequency intervals of the carriers. Therefore the wide-band interference can be dramatically mitigated. The steps of this mode are as follows:

STEP-1- Partition the UWB band into three bands: $\left(f_{L}, f I_{L}\right),\left(f I_{L}, f I_{H}\right)$, and $\left(f I_{H}, f_{H}\right)$. Then use the eigenvalue decomposition method to generate three sub-pulses $s_{I}(t), s_{2}(t)$, and $s_{3}(t)$ in each sub-band respectively. Note that $s_{2}(t)$ is in the interference bandwidth. So at the receiver, we can't use it before further processing.

STEP-2-Compose a new pulse $s(t)$ with $s_{1}(t), s_{2}(t), s_{3}(t)$

$$
s(t)=s_{1}(t)+s_{d}(t)+s_{3}(t)
$$

The spectrum of the new pulse is

$$
S(f)=S_{1}(f)+S_{\mathrm{d}}(f)+S_{3}(f)
$$

STEP-3- Based on the idea proposed in the two sub-bands mode, the received pulse in middle band $s_{2}(t)$ is processed in the same way. The processed pulse $s_{d}(t)$ can be expressed as:

$s_{d}(t)=(1 / 2)^{1 / 2}\left(s_{2}(t)-s_{2}\left(t-T_{g}\right)\right)$. And the spectral amplitude

of such a pulse can be computed as

$$
\left|S_{d}(f)\right|^{2}=2\left|S_{2}(f)\right|^{2} \sin ^{2}\left(\pi f T_{g}\right)
$$

Therefore, the spectrum has nulls at frequencies at $f=k T_{g}$ for any integer $k$. Suppose the wide-band system utilizes $N$ carriers, and the frequency interval between two carriers is $F_{I}$. We set $T_{g}=1 / F_{I}$. So we can remove all the interference on every carrier. Figure. 5 shows the PSD of the processed pulse in the middle band.

\section{INTERFERN SUPPRESION BASED ON TR-UWB SYSTEM USING AcR WITH NBI}

In this Section we look at the effects of a narrowband signal on the performance of the UWB TR-AcR. We find it convenient and reasonable to model the NBI at the UWB receiver front-end as a single tone sinusoidal signal[11]. Hence,

$$
i(t)=\sqrt{2 I} \cos \left(w_{i} t+\theta_{i}\right) * h_{N B}(t)
$$

represents the received interferer signal, with transmitted power equal to $I$, at the frequency fi $=2 \pi w_{i}$, with phase $\theta_{i}$, and after the frequency-flat fading channel $h_{N B}=\beta \delta\left(t-t_{0}\right)$, where $\beta$ is the channel gain and to the time shift. Note that the

equivalent baseband model is not used and the signal is real valued. We shall englobe the phase shift in the phase $\theta i$, which can be modelled as a random variable. uniformly distributed over the interval $[0 ; 2 \pi)$. Then,

$$
i(t)=\sqrt{2 I} \cos \left(w_{i} t+\theta_{i}\right)
$$

Figure. 1 shows the system model where UWB signals are received with a TR receiver in the presence of AWGN and interference from IEEE 802.11a WLAN systems. Out of band noise was removed using wideband band pass filter (BPF). It should be noted that in all computer simulations, the BER performance is based on assuming perfect synchronization and prior knowledge of the exact integration period. The decision variable at the AcR's output changes into

$$
\begin{aligned}
& z=\sum_{j=0}^{N_{d}-1} b_{j} \int_{j T_{d}}^{j T_{d}+T_{I}}[r(t)+i(t)]\left[r\left(t+D_{j}\right)+i\left(t+D_{j}\right)\right] d t \\
& =\varphi+n+\chi \\
& \quad \text { where } \chi \text { is the extra nuisance term due to the NB signal. }
\end{aligned}
$$

We can decompose it as the sum of three terms, i.e.

$$
\chi=\chi_{i}+\chi_{i i}+\chi_{n}
$$

Each one is given by

$$
\begin{aligned}
& \chi_{i}=\sqrt{E_{w}} \sum_{j=0}^{N_{d}-1} b_{j} \int_{0}^{T_{I}} g(t) i\left(t+j T_{d}+D_{j}\right) d t+ \\
& \sqrt{E_{w}} \sum_{j=0}^{N_{d}-1} b_{j} a_{j} \int_{0}^{T_{I}} g(t) i\left(t+j T_{d}\right) d t
\end{aligned}
$$

$\chi_{i i}=\sum_{j=0}^{N_{d}-1} b_{j} \int_{j T_{d}}^{j T_{d}+T_{I}} i(t) i\left(t+D_{j}\right) d t$

$\chi_{n}=\sum_{j=0}^{N_{d}-1} b_{j} \int_{j T_{d}}^{j T_{d}+T_{I}}\left[n(t) i\left(t+D_{j}\right)+i(t) n\left(t+D_{j}\right)\right] d t$

The last term $\chi_{n}$ represents an extra noisy-term due to the 'dirty' signal template, which is corrupted both from noise and NB signal.

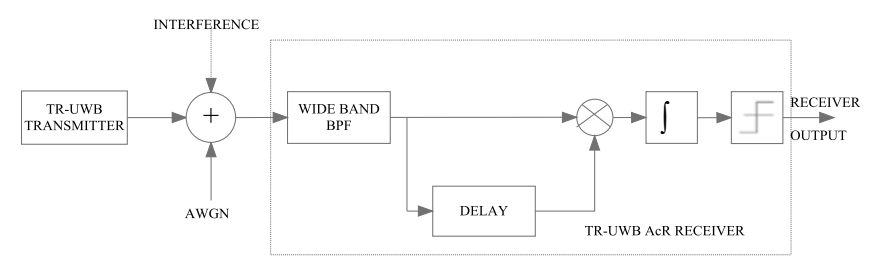

Figure 1. TR-UWB transceiver with channel consisting of AWGN and single narrowband interference. 


\section{SUPPRESSION OF INTERFERENCE FROM SINGLE NBI, MULTIPLE NBI AND WIDEBAND INTERFERENCE TO UWB SYSTEM USING NOTCH FILTER AT RECEIVER}

Interference saturates an unprotected UWB receiver front-end. Here, we introduce a simple method to suppress interference in TR receivers while preserving the desired UWB signal. Interference suppression in the TR receiver is achieved by eliminating the interfering band by a notch filter. Gaussian pulse has the advantage again, because notch filter bandwidth can be relatively narrowed compared to the full-band of Gaussian pulse. Notch filter effectively causes the interfering band to be "turned off". Notch filter is implemented with filter order of 400 and operates around $5.25 \mathrm{GHz}$. Also, it reduces interference to the UWB system from the multiple NBI and wideband interference. The block diagram of the modified TR receiver is shown in Figure. 2. A smart design of the chip and delay hoping code results in an effective suppression of the bias term $\chi_{i i}$. However, the performance degradation due to the remaining terms $\chi_{i}+\chi_{n}$ could still be unacceptable. The exact knowledge of the interferer frequency $\mathrm{f}_{\mathrm{i}}$ could be exploited to design a chip and delay hopping code aimed to minimize and thus $\chi_{i}$. Besides the obvious difficulty (if not impossibility) of obtaining the exact value of $f_{i}$, and of implementing accurate delay lines (the accuracy of the delay must be much smaller than $1 / \mathrm{f}_{\mathrm{i}}$ ), the suppression of $\chi_{n}$ remains unsolved. The best we could do is to cancel $i(t)$ before operating the correlation between received and delayed signal. Exploiting the (ultra-) large ratio between the UWB and NB signal, we are ready to sacrifice a fraction of the useful UWB signal energy in return of the suppression of the all NBI, by positioning a notch filter around the carrier frequency of the NB signal as shown in Figure 2.

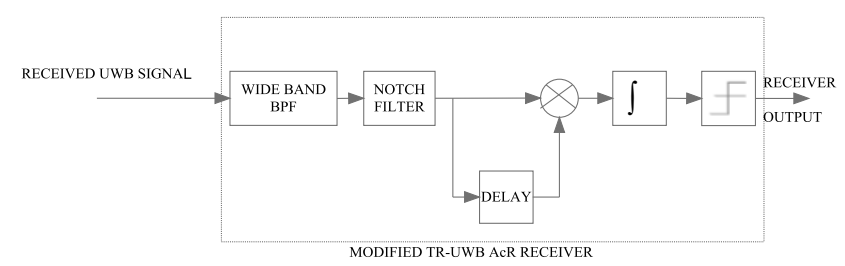

Figure 2. Block diagram of a modified TR receiver with a notch filter.

\section{SIMULATION STUDY AND ANALYSIS}

\subsubsection{UWB pulse design for single NBI, multiple NBI and wideband interference mitigation}
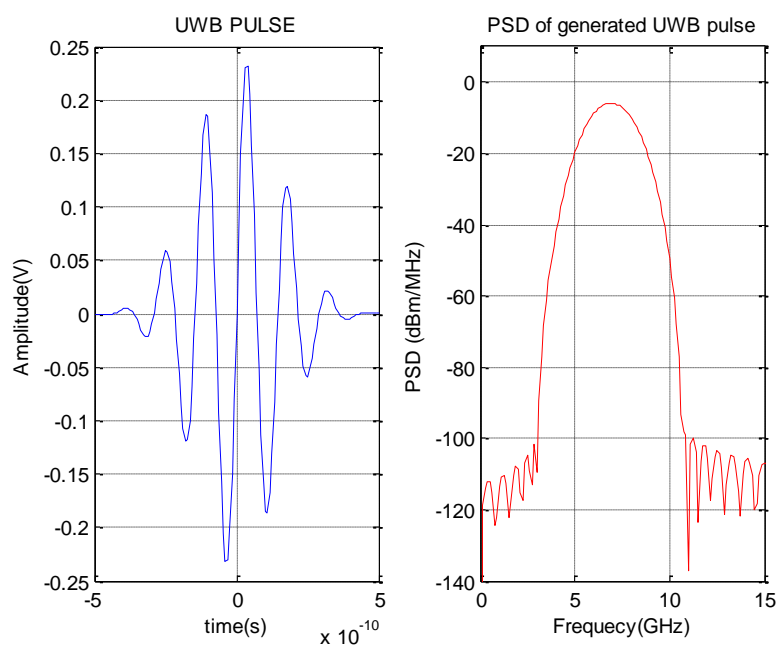

Figure 3. UWB pulse obtained from eigenvalue decomposition algorithm and PSD of generated UWB pulse
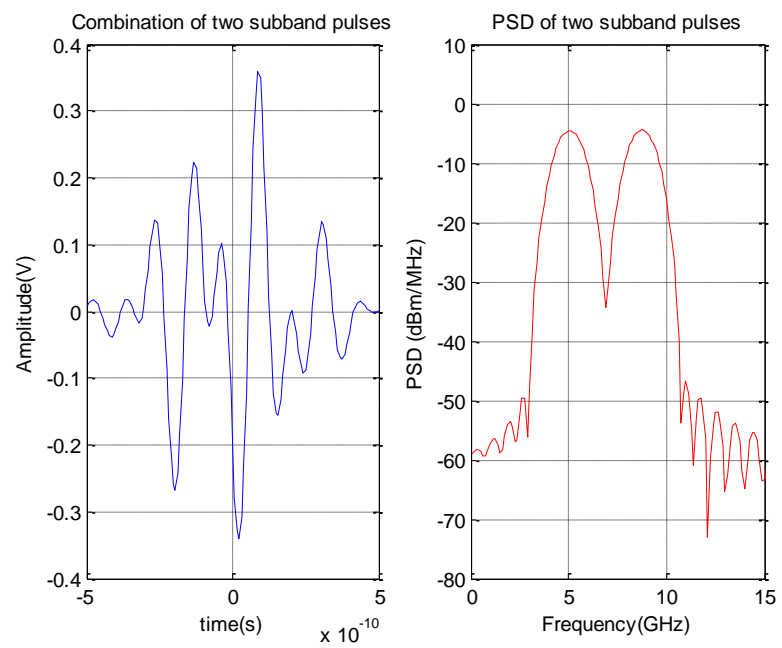

Figure 4. Combination of two sub-band pulses and PSD of combination of two sub-band pulses 

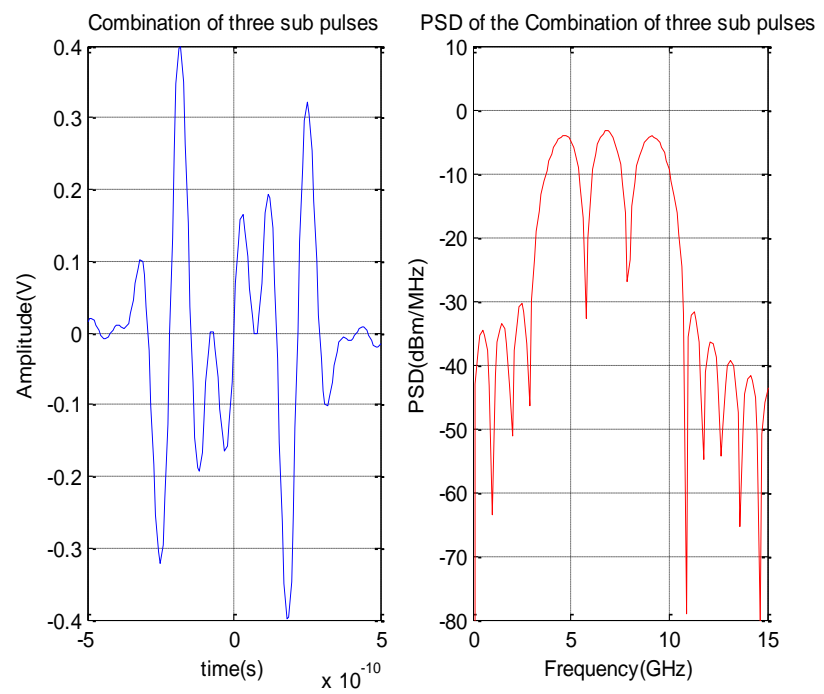

Figure 5. Combination of three sub-pulses and PSD of the combination of three sub-pulses

\subsection{2. $\quad$ BER Performance}

Figure. 6 illustrates a comparison of BER performance AcR TR receiver and a conventional TR receiver in a transmission channel consisting of a combined AWGN plus strong interference from single IEEE 802.11a WLAN interferer. By using wavelet based AcR TR receiver without interference we got a more than 6dB SNR improvement than that of AcR TR receiver with NBI at $10^{-2}$ BER. TR-UWB theoretical simulation provides better performance than all. For Figure. 7 a comparison takes place between improved AcR TR receivers. It means that coexistence different interference along with AcR TR receiver with notch filter is compared at the same BER floor.

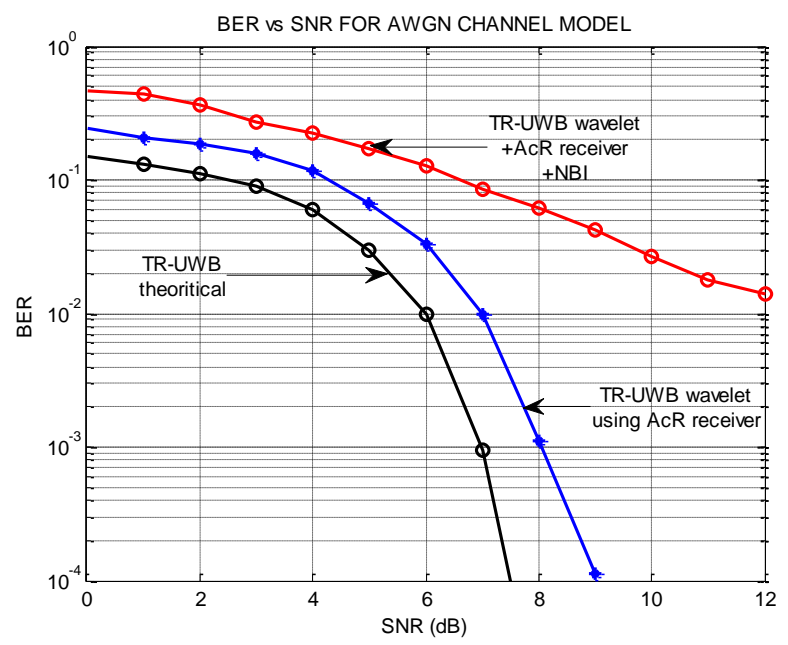

Figure 6. BER performance AcR TR receiver and AcR TR receiver with single $\mathrm{NBI}$

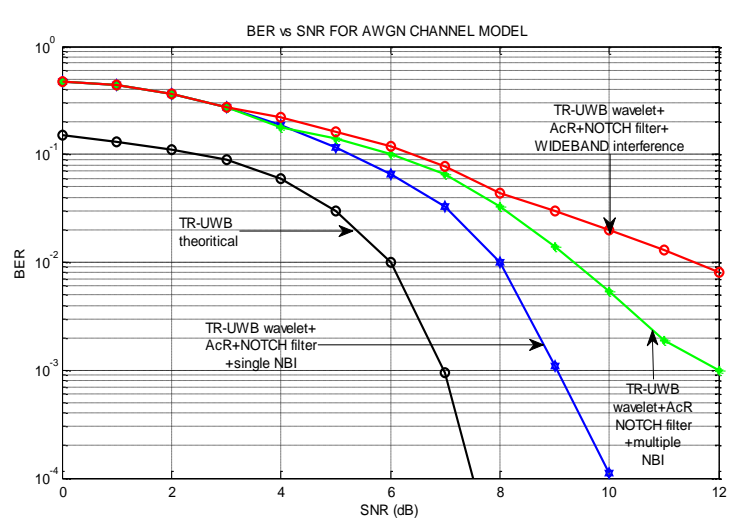

Figure 7. BER performance for different interference along with AcR TR receiver with notch filter

Figure. 6 and 7 provide AcR receiver with notch filter performs better than AcR receiver without notch filter for the same single NBI interference. Interesting result comes when multiple NBI and wideband interference is applied to AcR TR receiver with notch filter. The improved receiver with single NBI provides better performance than that of multiple NBI and wideband interference. Whereas multiple NBI with modified receiver performs a nearly $3 \mathrm{~dB}$ SNR improvement at $10^{-2} \mathrm{BER}$ floor than modified receiver with wideband interference.

\section{CONCLUSION}

So interference from UWB to other system by using pulse design technique has some advantages such as the pulses meets FCC spectral mask, occupies a short duration and easy to implement.TR-UWB system performance is studied in the presence of a strong single NBI interference. As shown, interference from TR-UWB to NBI is reduced by the energy in overlapping bands by using multicarrier based transmission pulses. Spectral density of the transmitted UWB signal around the interfering band is reduced than the peak. The BER performance of modified TR-UWB receiver outperforms that of conventional TR receiver. Also, the interference from single and multiple NBI to TR-UWB is reduced by a notch filter at the TRUWB receiver. Thus it can be concluded that this system provides very good technical solution to be used as UWB PHY layer for shortrange high data-rate wireless applications.

\section{REFERENCES}

[1] Federal Communications Commission, "New publicsafety applications and broadband internet access among uses envisioned by fcc authorisation of ultrawideband technology," FCC, 2002.

[2] D. Zeng, A. Annamalai Jr., and A.I. Zaghloul, "Pulseshaping filter design in uwb system," IEEE Conference on Ultra Wideband Systems and Technologies, Nov 2003, pp. 66-70.

[3] Y. Li and X.Huang, "The spectral evaluation and comparison for ultra-wideband signals with different modulation schemes," The 2000 World Multiconference on Systemics, July 2000, pp. 277-282. 
[4] R S DILMAGHNI, G MOHAMMAD, A BEN, "Novel UWB pulse shaping using prolate spheroidal wave functions," PIMRC2003,Beijing, 2003,pp.602-606.

[5] J J KNAB, "Interpolation of band-limited functions using the approximate prolate series," IEEE Trans Inform Theory (Corres), vol. IT-25, 1979, pp. 717-720.

[6] F. Dowla, F. Nekoogar, and A. Spiridon, "Interference mitigation in transmitted-reference ultrawideband(uwb) receivers," IEEE International Symposium on Antennas and Propagation, 2004, vol. 2, pp.1307-1310.

[7] K. Ohno, T. Ikebe, and T. Ikegami, "A proposal for an interference mitigation technique facilitating the coexistence of bi-phase uwb and other wideband systems," IEEE IWUWBS UWQBST 2004, May 2004, pp. 50-54.

[8] J. Han and C. Nguyen, "A new ultra-wideband, ultra-short monocyclepulse generator with reduced ringing," IEEE Microwave Wireless Compon.Lett., vol. 12, pp.206-208, June 2002.
[9] Taha., K. M. Chugg, "A theoretical study on the effects of interferenceUWB multiple access impulse radio Signals," Systems and Computers2002 Conference Record of the ThirtySixth Asilomar Conference.,Vol.1,pp.728-732,Nov2002.

[10] Sablatash M, Sellathurai M. "Methods for interference mitigation by and into UWB communication systems, including techniques based on multi-band techniques and on wavelets." In: Proceedings of the 22nd biennial symposium on communications, Kingston, Canada, May 2004.

[11] Q. Dang, A. Trindade, A.-J. van der Veen, and G. Leus, "Signal model and receiver algorithms for a transmit-reference ultrawideband communication system," IEEE Journal on Communications,vol. 24, no. 4, pp.773-779, April 2006.

[12] Zhendong Luo, Hong Gao, Yuanan Liu, and Jinchun Gao" A New UWB Pulse Design Method for Narrowband Interference Suppression" IEEE Communications Society, Globecom 2004 